\title{
Guide-wire fragment embolisation in paediatric peripherally inserted central catheters
}

\section{Joel M Dulhunty MB BS, MTH, PhD, Medical Administration Registrar,' and Research Fellow, Burns, Trauma and Critical Care Research Centre ${ }^{2}$ \\ Andreas Suhrbier BA, PhD, NHMRC Principa Research Fellow}

Graeme A Macaulay BEng(Med), Principal Biomedical Engineer ${ }^{4}$

Jennifer C Brett BEng(Med) Biomedical Engineer ${ }^{4}$

Alexa V A van Straaten BA(Hons), MA, MPhil, Principal Project Officer, Patient Safety Notifications: Medical Devices

Ian M Brereton $\mathrm{BSc}, \mathrm{PhD}$ Director for Research and Technology, Centre for Advanced Imaging ${ }^{2}$

Jillann F Farme MB BS, FRACGP, FRACMA Medical Director and Adjunct Associate Professor of Psychology ${ }^{2}$

1 Patient Safety and Quality Improvement Service

Queensland Health Brisbane, QLD.

2 University of Queensland Brisbane, QLD.

3 Queensland Institute of Medical Research,

Brisbane, QLD.

4 Biomedical

Technology Services,

Queensland Health,

Brisbane, QLD.

jillann_farmer@

health.qld.gov.au

MJA 2012; 196: 250-255 doi: 10.5694/mjal2.10097

Editorial p 222

Perspective p 232

Letter $p 240$

Research p 256
$+$ ach year, over 1000 medical device incidents are reported to the Australian Therapeutic Goods Administration (TGA). The most recent published statistical reports from this scheme show 926 reports in 6 months (January-June 2009), of which 597 resulted in serious injury and 25 were associated with patient death. ${ }^{1,2}$

The 3 Fr peripherally inserted central catheter (PICC) is a medical device commonly used in infants and children for administration of medication and fluids, and for blood sampling. ${ }^{3}$ Reported complication rates range from zero to $33.6 \%{ }^{4}$ with occlusion and catheter-related bloodstream infection being the most common. Rarer complications include phlebitis, venous or right atrial perforation and extravasation, pleural effusion, pericardial effusion or tamponade, dysrhythmias and mechanical failure, such as leakage, migration of the tip, and line fracture. ${ }^{4,5}$ Although line fracture and embolisation have been studied in children and adults, ${ }^{5}$ guide-wire fragment embolisation has been confined to a single case report. 6

This article comprises four parts: a case series report of 3 Fr PICC guidewire fragment embolisations, a clinician user-experience survey, a device design evaluation, and a magnetic resonance imaging (MRI) simulation study. The latter three elements directly compare safety performance of a range of 3 Fr PICC products.

\section{Methods}

\section{Clinical incidents}

After a cluster of three embolisation events in 2008, we undertook a 2-year retrospective review. File reviews,

\section{Abstract}

Objective: To report guide-wire fragment embolisation of paediatric peripherally inserted central catheter (PICC) devices and explore the safety profile of four commonly used devices.

Design, setting and participants: Clinical incidents involving paediatric PICC devices in Queensland public hospitals were reviewed. A PICC user-experience survey was conducted at five public hospitals with 32 clinicians. A device design evaluation was undertaken, and magnetic resonance imaging (MRI) safety was tested by a simulation study.

Main outcome measures: Embolisation events; technical mistakes, multiple attempts and breakages during insertion; willingness to use the device; failure modes and risk priority rating; movement and/or temperature change on exposure to MRI.

Results: Six clinical incidents of silent guide-wire embolisation, and four near misses were identified; all were associated with one device. The survey found that this device had a reported broken-wire embolisation rate of 0.9/100 insertions with no events in other devices; two of the four devices had a higher all-cause embolisation rate (3.3/100 insertions $v 0.4 / 100$ insertions) and lower clinician acceptance (68\%-71\% v 91\%-100\%). All devices had 6-17 identified failure modes; the two devices that allowed removal of a guide wire through a septum had the highest overall risk rating. Guide-wire exposure to MRI was rated a potential safety risk due to movement.

Conclusions: There is marked variation in the safety profile of $3 \mathrm{Fr} \mathrm{PICC}$ devices in clinical use, and safety performance can be linked to design factors. Pre-MRI screening of all children who have previously had a PICC device inserted is recommended. We advocate a decision-making model for evaluation of device safety.

clinical incident data and a government hotline were used for case-finding. Children (aged < 16 years) who had received a PICC in the preceding 2 years at a Queensland public hospital were identified. Existing postinsertion chest $x$-rays were reviewed for evidence of a retained wire, and new films taken where necessary. This was a statewide review, with hospitals reporting their findings centrally.

\section{Clinician user-experience survey}

Clinicians with experience in inserting paediatric PICC devices were invited to complete an anonymous online survey between 27 April and 19 September 2010. Recruitment was by email invitation at five tertiary public hospitals, which had collectively undertaken $62 \%$ of the insertions identified in the retrospective review:
Townsville Hospital, Gold Coast Hospital, Mater Children's Hospital, Royal Children's Hospital (Brisbane) and the Prince Charles Hospital. A reminder was sent 7-10 days before survey closure.

The survey contained 11 questions about frequency of insertion, past experience, user preferences and respondent demographics. Participants were asked to rate the frequency of the following events for each of the four PICC devices and for any other $3 \mathrm{Fr}$ PICCs used: the number of insertions, technical mistakes, multiple insertion attempts, total-wire embolisation, broken-wire embolisation, and line fracture with embolisation. Participants were asked about ease and intuitiveness of use for each device, and to rate their willingness to use the product in the future. 


\section{Device design evaluation}

Devices were evaluated in a simulated clinical environment using a modified failure mode and effects analysis (FMEA) methodology. ${ }^{7} \mathrm{~A}$ "failure mode" is the way in which a device can fail.

A group comprising two doctors (JFF and a paediatric intensivist), and two biomedical engineers (GA M and J CB) determined the potential failure modes for each device.

The occurrence rating $(\mathrm{O})$ is the probability that the failure mode will occur. The potential resultant harm to the patient is measured with the severity rating $(\mathrm{S})$. The probability that the failure mode will actually result in harm is recorded as a likelihood rating (L). Occurrence and likelihood use the same descriptors, but measure different events (Box 1 ).

The risk priority number (RPN) is the product of the occurrence, severity and likelihood ratings $(\mathrm{O} \times \mathrm{S} \times \mathrm{L})$, and is used to compare the level of risk associated with each of the failure modes. For each device, failure modes for each step of use were identified and RPNs calculated.

\section{Magnetic resonance imaging safety study}

The theoretical MRI safety risk associated with heating and movement of an embolised wire fragment was evaluated. ${ }^{8}$ The ferromagnetic properties of the wires were confirmed by exposure to a magnet. A straight $(7 \mathrm{~cm})$ and looped $(20 \mathrm{~cm})$ wire fragment from each device was partially embedded in agar gel $(1.5 \mathrm{~g}$ agar in $100 \mathrm{~mL}$ phosphate-buffered saline) in an $88 \mathrm{~mm}$ Petri dish with graph paper on the underside. Movement was detected by direct and video observations during: (1) entry into the static magnetic field of a Magnetom Trio Tim 3T MRI scanner (Siemens Healthcare, Erlangen, Germany) using syngo MR software (Siemens Healthcare); (2) 7 minutes of scanning using TrueFISP (Siemens Healthcare) (sequence trfi2d1_43, repetition time [TR] $171 \mathrm{~ms}$, echo time [TE] $1.3 \mathrm{~ms}, 26$ slices, 60 degree pulse angle); and (3) rotation of the plate in a static $3 \mathrm{~T}$ MRI field to simulate patient movement. Temperature of the wire-gel interface was measured with a thermocouple probe (Digitech thermometer, Electus Distributions, Sydney, NSW) before and after exposure to the MRI field. Potential MRI risk was identified by

\section{Rating scales used in the failure mode and effects analysis}

\section{A. Occurrence and likelihood ratings}

Occurrence and likelihood rating

\begin{tabular}{ll}
\hline 1: rare & The event may occur only in exceptional circumstances \\
2: unlikely & The event is not expected to occur \\
3: possible & The event might occur at some time \\
4: likely & The event will probably occur on multiple occasions \\
5: almost certain & The event will occur in most circumstances
\end{tabular}

\section{B. Severity ratings}

Severity rating Adverse clinical outcome Adverse effect on device

$\begin{array}{lll}\text { 1: negligible } & \begin{array}{l}\text { No injury or harm caused, minor adjustment to } \\ \text { operational routine }\end{array} & \text { No impact on function of medical device } \\ \text { 2: minor } & \text { Minimal harm caused, slight interruption to routine } & \text { Minor impact on function of medical device } \\ \text { 3: moderate } & \text { Loss of function, major harm caused } & \text { Moderate impact on function of medical device } \\ \text { 4: major } & \text { Loss of life } & \text { Major impact on function of medical device } \\ \text { 5: extreme } & \text { Multiple deaths } & \text { Cessation of function of medical device }\end{array}$

ferromagnetism with either movement of the wire, or a temperature rise of $>3.0^{\circ} \mathrm{C}$ measured next to the wire. ${ }^{9}$

\section{Ethics approval}

Ethics approval was obtained from the Metro South Health Service District Human Research Ethics Committee, and from each of the five facilities where the clinician survey was conducted.

\section{Results}

\section{Clinical incidents}

The sentinel event in this case series was the incidental identification of a fine filament of wire on chest $x$-ray after insertion of a 3 Fr PICC in a 4-year-old child in June 2008. The outer coiled covering of the guide wire had delaminated and separated from the inner core. Computed tomography confirmed a metallic wire within the right ventricle and extending into a pulmonary artery. The embolised wire was removed by cardiac catheterisation.

In October 2008, two further embolisation events were identified at the same facility in children who had received the same type of PICC device. A local review identified a further two cases of radiologically confirmed guide-wire embolisation involving the same device.

Each of the five cases identified was undetected at the time of insertion. As

\section{Embolised wire fragment on chest $x$-ray, supine (A) and lateral $(B)$ views}

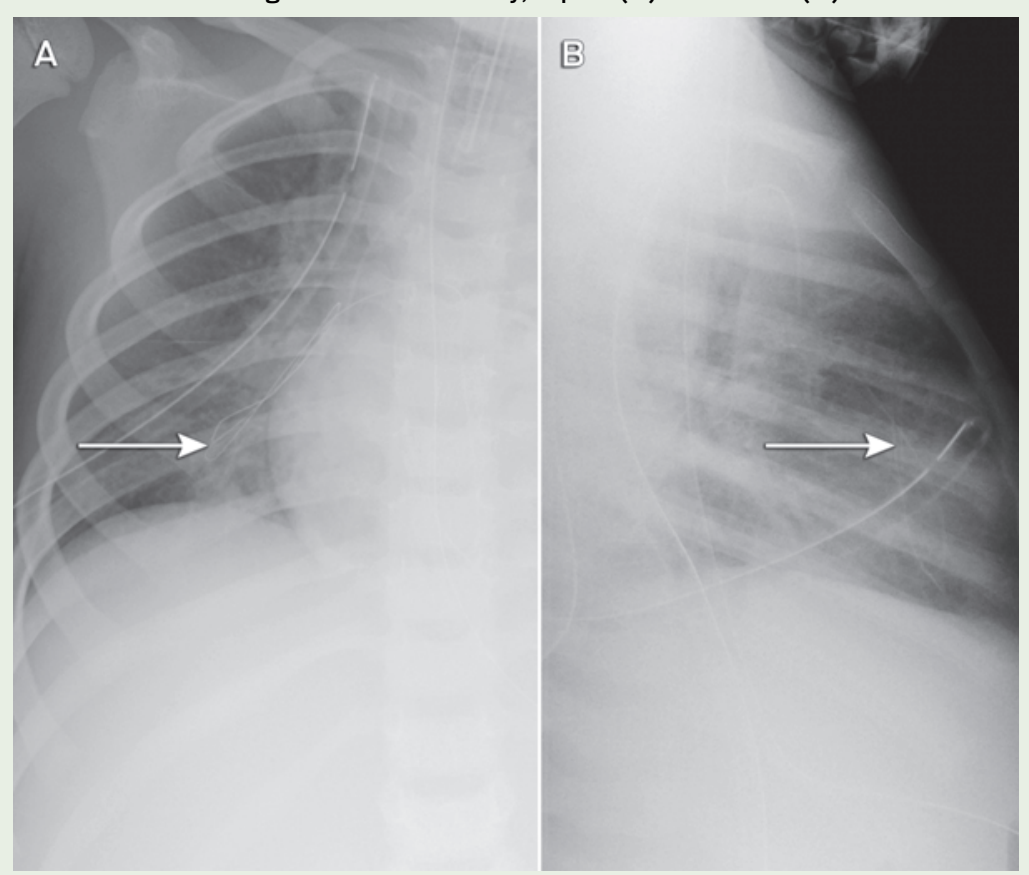


3 Results of a user survey of 32 clinicians at five Queensland hospitals, 2010

\begin{tabular}{|c|c|c|c|c|}
\hline Characteristic & $\begin{array}{c}\text { Device A, no. } \\
(\%)\end{array}$ & $\begin{array}{l}\text { Device B, no. } \\
(\%)\end{array}$ & $\begin{array}{l}\text { Device C, no. } \\
(\%)\end{array}$ & $\begin{array}{c}\text { Device D, no. } \\
(\%)\end{array}$ \\
\hline Practitioners & $24(75 \%)$ & $9(28 \%)$ & $25(78 \%)$ & $15(47 \%)$ \\
\hline Estimated total insertions* & 218 & 208 & 548 & 249 \\
\hline \multicolumn{5}{|l|}{ Embolisation events $^{\dagger}$} \\
\hline Broken-wire embolisation & $2(0.92 \%)$ & 0 & 0 & 0 \\
\hline Line fracture and embolisation & $6^{\ddagger}(2.75 \%)$ & $6^{\ddagger}(2.88 \%)$ & $3(0.55 \%)$ & 0 \\
\hline Total events & $8(3.67 \%)$ & $6(2.88 \%)$ & $3(0.55 \%)$ & 0 \\
\hline \multicolumn{5}{|l|}{ Usability } \\
\hline \multicolumn{5}{|l|}{ Technical mistakes with insertion ${ }^{\S}$} \\
\hline$\geqslant 1$ in 10 & $3(13 \%)$ & 0 & $1(4 \%)$ & 0 \\
\hline$<1$ in 10 & $21(88 \%)$ & $9(100 \%)$ & $24(96 \%)$ & $15(100 \%)$ \\
\hline \multicolumn{5}{|l|}{ Multiple insertion attempts ${ }^{5}$} \\
\hline$\geqslant 1$ in 10 & $14(58 \%)$ & $2(22 \%)$ & $14(56 \%)$ & $5(33 \%)$ \\
\hline$<1$ in 10 & $10(42 \%)$ & $7(78 \%)$ & $11(44 \%)$ & $10(67 \%)$ \\
\hline \multicolumn{5}{|l|}{ Breakages during insertion ${ }^{\varsigma}$} \\
\hline$\geqslant 1$ in 10 & $3(13 \%)$ & 0 & $2(8 \%)$ & 0 \\
\hline$<1$ in 10 & $21(88 \%)$ & $9(100 \%)$ & $23(92 \%)$ & $15(100 \%)$ \\
\hline Total $(\geqslant 1$ in 10$)$ & $20(83 \%)$ & $2(22 \%)$ & $17(68 \%)$ & $5(33 \%)$ \\
\hline Willingness to useq & $15 / 22(68 \%)$ & $5 / 7(71 \%)$ & $21 / 23(91 \%)$ & 14/14 (100\%) \\
\hline
\end{tabular}

* Estimated total insertions were calculated using the mid-range value of ordinal categories ( 3 for $1-5,13$ for $6-20,36$ for $21-50,76$ for $51-100$ and 101 for $>100$ ) multiplied by the number of practitioners reporting usage in that range. $†$ Percentage embolisation (total wire, broken wire and line fracture) was calculated using the estimated total insertions in the denominator. $\ddagger$ Rated as $>5$ by two participants. $§$ Percentage of technical mistakes, multiple insertion attempts and breakages during

insertion was calculated using number of practitioners in the denominator. qWillingness to use was dichotomised as "very/quite

happy to use" versus "will use if no alternative/will not use" with number of practitioners reported in the denominator.

only part of the guide wire embolised, the portion withdrawn by the operator provided a false reassurance. The extremely fine embolised wires had not been noted on post-insertion chest $\mathrm{x}$-rays (where the focus is usually on the position of the catheter tip), but were apparent when a specific review of the films was undertaken (Box 2). This cluster of five cases (involving different operators) at a single hospital triggered a statewide case review, through which an additional five cases in two further hospitals were reported (one actual embolus and four "near misses", where the wire unravelled, but was noticed and retrieved before entering the patient). All involved the same type of device, giving a total of 10 cases at three hospitals out of 710 patients followed up. Although objective data about whether the wire was cut in every case were not available (because it can be cut without detection), at least one clinician was certain the wire had not been cut.

The reported event rate of wirefragment embolisation was 0.84 per 100 patient insertions. Adding the near-miss events gives an overall reported event rate of 1.4 per 100 patient insertions.

Initial evaluation suggested potential contributing factors including product design (a trimmable PICC with a fine-gauge wire preloaded through a constrictive septum with the potential for shearing if withdrawn), latent factors (insufficient instructions for use with inadequate warnings about not trimming the wire or withdrawing the guide wire through the septum) and human factors (eg, withdrawal of the guide wire through the septum). Only one set of instructions was available in each box of five catheters. An investigation by the TGA, which concluded in November 2008, required improvements to label warnings and user instructions, but found no inherent flaw with the PICC devices. ${ }^{10}$

\section{Clinician survey of peripherally inserted central catheter usage}

The clinician survey examined user experience. Of the 55 respondents, 39 had previously inserted a 3 Fr PICC device. Information on complications, including the frequency of technical mistakes, need for multiple attempts and breakages associated with insertion, was completed by 32 respondents (Box 3). Of these, there were 23 medical practitioners, eight nurses and one sonographer.

Five practitioners (16\%) had used all four devices, eight practitioners (25\%) had used three devices, 10 (31\%) had used two devices, and nine (28\%) had used only one of the devices. No other types of PICC devices were used. Two broken-wire embolisation events were reported for the same type of device as in the original case series, but not for any of the other devices (Box 3).

The reported event rate in the clinician survey for wire fragment embolisation was 0.9 in 100 insertions for one device and 0.16 in 100 insertions across all devices.

\section{Device design evaluation}

The device design evaluation assessed the latent safety features of the four devices, including design features

\section{Summary of failure mode and effects analysis results}

\begin{tabular}{|c|c|c|c|c|c|c|c|c|}
\hline \multirow[b]{2}{*}{ Function } & \multicolumn{2}{|c|}{ Device A } & \multicolumn{2}{|c|}{ Device B } & \multicolumn{2}{|c|}{ Device C } & \multicolumn{2}{|c|}{ Device D } \\
\hline & $\begin{array}{l}\text { FM, } \\
\text { no. }\end{array}$ & $\begin{array}{c}\text { Mean RPN } \\
(\max )^{*}\end{array}$ & $\begin{array}{l}\text { FM, } \\
\text { no. }\end{array}$ & $\begin{array}{l}\text { Mean RPN } \\
(\max )^{*}\end{array}$ & $\begin{array}{l}\text { FM, } \\
\text { no. }\end{array}$ & $\begin{array}{c}\text { Mean RPN } \\
(\max )^{*}\end{array}$ & $\begin{array}{l}\text { FM, } \\
\text { no. }\end{array}$ & $\begin{array}{l}\text { Mean RPN } \\
(\max )^{*}\end{array}$ \\
\hline Catheter preparation & 4 & 29 (75) & 3 & $31(75)$ & 1 & $25(25)$ & 3 & $50(75)$ \\
\hline Catheter insertion & 3 & $19(48)$ & 3 & $21(48)$ & 2 & $30(48)$ & 2 & $16(16)$ \\
\hline Removal of guide wire & 9 & $51(64)$ & 3 & $32(48)$ & 2 & $24(36)$ & 0 & na \\
\hline Finalisation and use & 1 & $75(75)$ & 1 & $75(75)$ & 1 & $75(75)$ & 3 & $35(48)$ \\
\hline Total & 17 & $174(262)$ & 10 & $159(246)$ & 6 & $154(184)$ & 8 & 101 (139) \\
\hline
\end{tabular}

$\mathrm{FM}=$ failure mode. $\max =$ maximum. RPN = risk priority number, calculated as the product of occurrence, severity and likelihood of the failure mode causing harm. na $=$ not applicable. $*$ The mean and maximum RPNs are reported for the various FMs related to each function. 


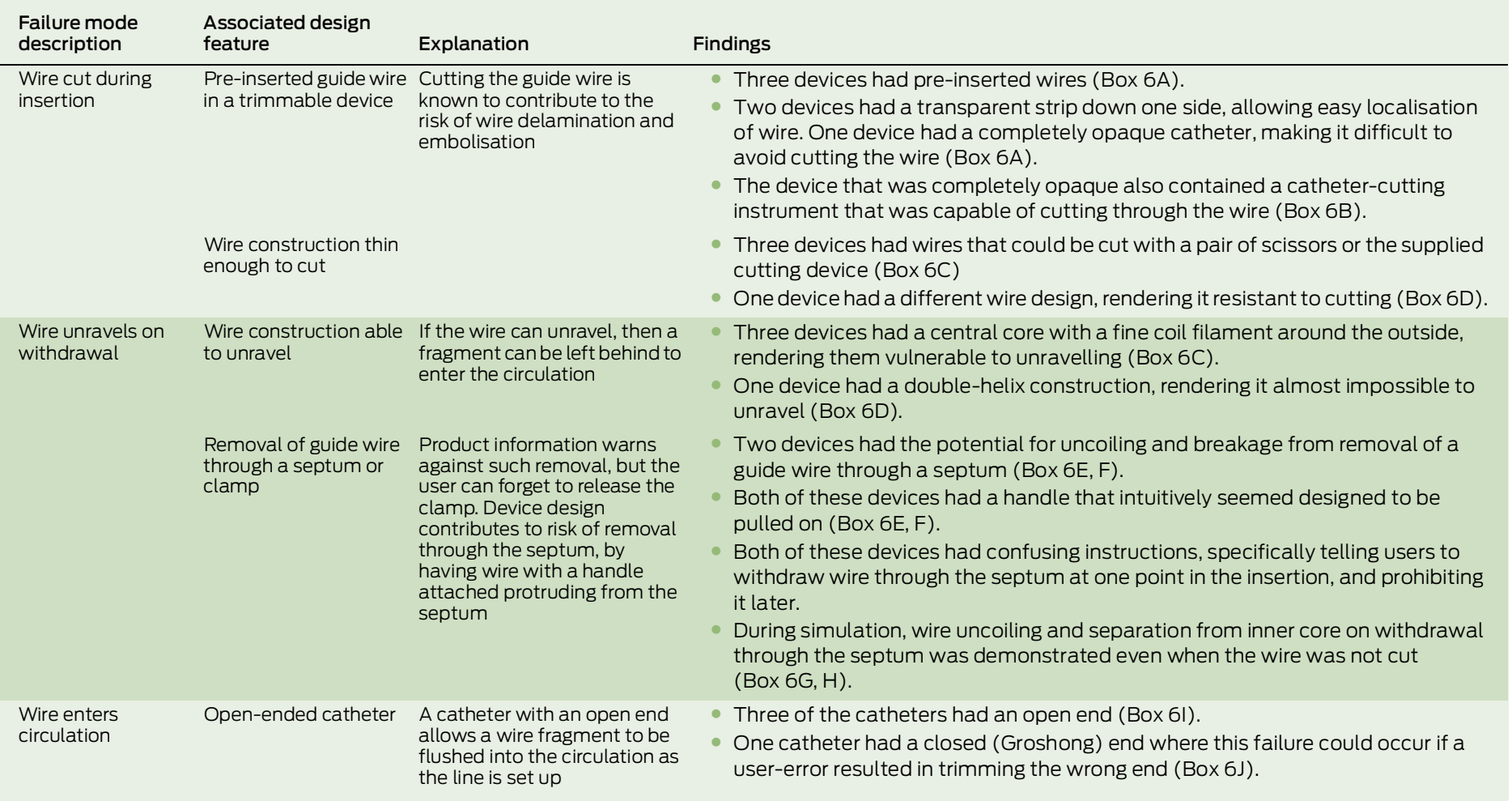

resistant to clinician error. A range of six to 17 potential design failure modes were demonstrated across each of the four devices (Box 4). Failure modes for several clinically relevant complications were linked to five key design elements (Box 5, Box 6). The presence of all five design elements increased the risk of wire fragment embolisation.

\section{Magnetic resonance imaging safety study}

A potential risk of an undetected wire fragment in situ was exposure to MRI resulting in tissue and thermal injury. ${ }^{11}$ All four guide wires were ferromagnetic when exposed to a magnet. On entrance into the MRI field, all wire types moved with sufficient force to pull the partially embedded wire from the agar gel and unravel the coiled wire loop. Once the plate was positioned in the centre of the MRI magnet, wire movement was not observed with application of field gradients. Gentle rotation of the plate in the centre of the scanner resulted in reorientation of all four wire types in the direction of magnetic field lines. The median temperature change in the agar gel next to the wire was $1.5^{\circ} \mathrm{C}$ (range, $-0.9^{\circ} \mathrm{C}$ to $+1.8^{\circ} \mathrm{C}$ ) with a tem- perature rise of $0.5^{\circ} \mathrm{C}$ in a control plate without a wire fragment. All devices were rated as being of potential MRI risk due to possible movement and damage to surrounding tissue.

\section{Discussion}

In this study, we found that there is marked variation in the safety profile of four paediatric PICC devices in clinical use, and that this difference can be directly linked to defined design features.

\section{Implications for patient management}

The case series demonstrates that wire fragment embolisation can occur silently, and was observed in only one type of device in the case reviews and survey.

There is no other published safety information on PICC guide wires exposed to a magnetic field. On the basis of this study, we recommend that children who have had a PICC inserted need adequate screening before MRI.

Most PICC placements are checked on chest $x$-ray after insertion, so use of a sufficiently wide field and careful scrutiny to exclude silent embolisation is recommended as part of the routine post-insertion review. In children with a history of PICC insertion who require MRI, $x$-ray screening should be undertaken. To minimise radiation dose, this should be a review of the post-insertion films, which will usually be of sufficient quality to confidently exclude wire embolisation. If there is doubt, and if the PICC line inserted was of the design associated with embolisation risk in this study, specific pre-MRI screening should be undertaken.

If a wire is found, assessment for the risk of removal versus retention must be undertaken. Alternative imaging methods to MRI should be considered if the wire fragment remains in situ.

\section{Implications for device selection}

Our study establishes clear differences in the safety profile of four 3 Fr PICC devices. The device with all the "at risk" design elements had a higher incidence of guide-wire embolisations and near misses, with multiple incidents across three hospitals involving separate, experienced users.

Design factors clearly make a difference to safety and should influence decision making. Although safety is not 
6 Illustrations of design features relevant to failure modes described in Box 5
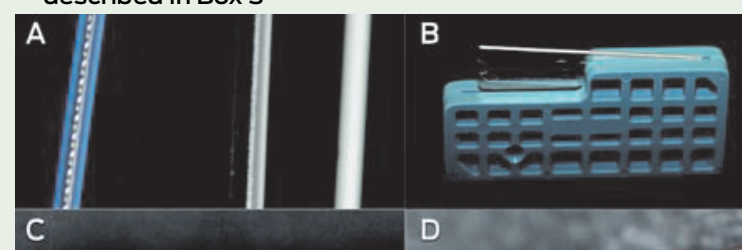

D
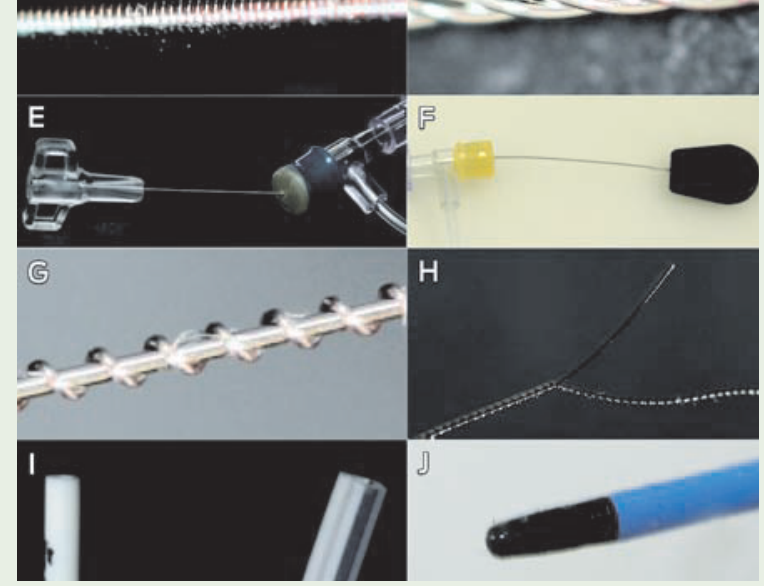

A. Peripherally inserted central catheters (PICCs) with preinserted wires. The two on the left are transparent on one side, allowing visualisation of guide wire. The one on the right is opaque throughout its circumference, preventing visualisation of the guide wire. $\boldsymbol{B}$. Catheter-cutting instrument included in the packet of the device with the opaque catheter. C. PICC guide wire with coil construction, which can be cut easily and is vulnerable to unravelling D. PICC guide wire with double-helix construction, which cannot be easily cut and is resistant to unravelling. E, F. PICC assemblies showing guide wire protruding from septum with handle attached to guide wire. G. Outer coil of guide wire uncoiling and stretching along the inner core after withdrawal through septum (wire not cut). H. Outer coil of guide wire completely separating from inner core. The outer coil is the component that breaks away and embolises. I. Open-ended catheter allows guide-wire fragment to migrate into vascular system. J. Closed (Groshong) end.

the sole factor in purchasing decisions, it must be considered. Devices do not necessarily have a similar safety profile simply because they are approved for marketing. "Fitness for purpose" (the regulator decision) is not the same as comparative safety evaluation.

Although warning labels and product information are sufficient to achieve TGA approval, their effectiveness is weakened by their interplay with human factors. Latent design features that support safety (eg, the closed end and double-helix construction of the wire) and "forcing functions" (design features that force users down the correct usage pathway) are more reliable than complex instructions. ${ }^{12}$ Reliance on clinician

7 Decision-making model for evaluating and managing medical device safety concerns

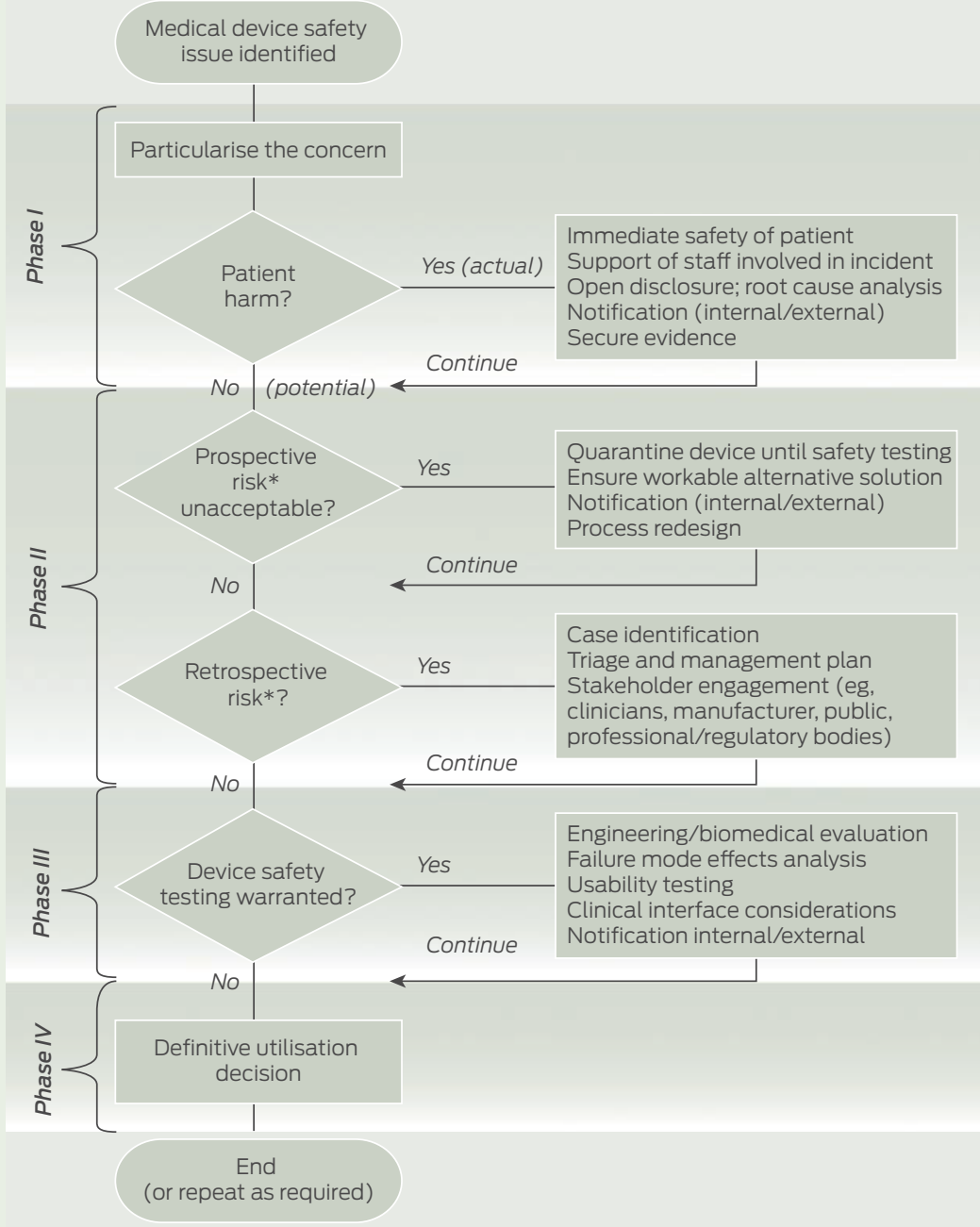

* To evaluate prospective risk, ask the question: "If we continue to use this device are future patients at risk of harm?" Retrospective risk involves considering whether patients previously exposed to the device are at current or future risk of harm.

vigilance and memory, in an environment of complexity or lack of standardisation, fails to acknowledge the propensity for human error.

\section{Implications for evaluating and managing safety concerns}

There is no standardised, published methodology for approaching this type of safety issue. Through the lessons learned in this case series and the subsequent device evaluation, we have developed a decision-making model for evaluating and managing similar safety concerns (Box 7).

Although the clinician survey is limited by unknown representativeness of the sample and the potential for recall bias, it demonstrates the benefit of combining experimental and experiential information in making device determinations. Risk-benefit considerations ultimately need to be made at several levels (eg, at the clinician/patient level, at the purchasing/ health care system level and at the regulatory level). Our results provide evidence to inform this decision-making process, particularly at the clinician and health care levels.

Our study demonstrates that not all 3 Fr PICC devices have the same safety profile. Clinicians and purchasing decisionmakers need to be aware of risks associated with medical devices to make decisions in the best interest of patients. When concerns are raised about a device, we advocate an approach to patient safety that comprises clinical management, risk management, device safety testing and a definitive utilisation decision. 
Acknowledgements: We acknowledge the staff of the Townsville Hospital who first identified and reported this issue. We thank Dr John Wakefield for his leadership in managing the original case series in Queensland, and for his introduction of the FMEA method to clinical incident management in Queensland. Mellissa Naidoo and Kirsten Price provided assistance with obtaining ethics approval for the survey. Kevin McCaffery provided assistance with the FMEA and with obtaining ethics approval for the survey. Thank you to Joy Gardner and Wayne Schroder for laboratory assistance with the MRI safety study. Gail Durbridge and Donald Maillet helped design and conduct the MRI safety study; David Lloyd helped conduct the MRI safety study. Madeleine Kersting took photographs of the devices. Joan Curtis provided administrative support Thank you to staff who assisted with obtaining devices for testing, participated in the survey and were involved in the notification, management and review of PICC-related incidents.

Competing interests: No relevant disclosures.

Received 15 Jan 2012, accepted 14 Feb 2012.

1 Therapeutics Goods Administration. Medical device incident reports 01/04/2009 - 30/06/ 2009. Canberra: TGA, 2009. http://www.tga. gov.au/hp/iris-articles-statistics-09040906.htm (accessed Feb 2012).
2 Therapeutics Goods Administration. Medical device incident reports 01/01/2009 - 31/03/ 2009. http://www.tga.gov.au/hp/iris-articlesstatistics-0901-0903.htm (accessed Feb 2012).

3 Knue M, Doellman D, Jacobs BR. Peripherally inserted central catheters in children: a survey of practice patterns. J Infus Nurs 2006; 29: 28-33.

4 Pettit J. Assessment of infants with peripherally inserted central catheters: Part 1. Detecting the most frequently occurring complications. $A d v$ Neonatal Care 2002; 2: 304-315.

5 Pettit J. Assessment of infants with peripherally inserted central catheters: Part 2. Detecting less frequently occurring complications. Adv Neonatal Care 2003; 3: 14-26.

6 Ragg P. Unravelling and embolisation of guidewires with PICC lines, March 2007. Melbourne: Australian and New Zealand College of Anaesthetists, 2007. http://www.anzca.edu. au/quality-safety/articles/archive/unravellingand-embolisation-of-guide-wires-with-picclines.html (accessed Feb 2012)

7 Institute for Healthcare Improvement. Failure Modes and Effects Analysis tool. Cambridge, Mass: IHI, 2011. http://www.ihi.org/knowledge/
Pages/Tools/FailureModesandEffectsAnalysis Tool.aspx (accessed Feb 2012).

8 Shellock FG, Woods TO, Crues JV, 3rd. MR labeling information for implants and devices: explanation of terminology. Radiology 2009; 253 : 26-30.

9 Shellock FG. The List: information and terminology. Los Angeles: MRIsafety.com, 2010. http://www.mrisafety.com/list.asp (accessed Feb 2012).

10 Therapeutics Goods Administration. Serious adverse events with the use of peripherally inserted central catheters (PICC lines). Canberra: TGA, 2008. http://www.tga.gov.au/safety/ alerts-device-picc-081113.htm (accessed Feb 2012).

11 Food and Drug Administration. A primer on medical device interactions with magnetic resonance imaging systems. Silver Spring, Md: FDA, 1997. http://www.fda.gov/MedicalDevices/ DeviceRegulationandGuidance/Guidance Documents/ucml07721.htm (accessed Feb 2012).

12 Norman DA. The design of everyday things. New York: Basic Books, 2002. 